\title{
Phase-Contrast OCT Imaging of Transverse Flows in the Mouse Retina and Choroid
}

\author{
Jeff Fingler, ${ }^{1}$ Carol Readhead, ${ }^{2}$ Daniel M. Schwartz, ${ }^{3}$ and Scott E. Fraser ${ }^{1,2}$
}

Purpose. To test the hypothesis that a novel phase-contrast optical coherence tomography (OCT) system can image retinal and choroidal vessels in the living mouse.

Methods. A high-speed spectral domain optical coherence tomography (SDOCT) system, which measures the reflections for the entire depth of the retina at once with each axial scan (A-scan), was developed for mouse retinal imaging. Acquiring multiple A-scans over a transverse line across the mouse retina offers a two-dimensional cross-sectional image (B-scan); several neighboring B-scans can be assembled into a three-dimensional OCT image. To visualize mobility and transverse flow in retinal vessels, the statistical variance of phase for each location was calculated from multiple B-scans acquired successively for the same retinal cross-section. Such measures of phase variance offer a direct measure of motions over a large dynamic range of flow velocities.

RESULTS. Three-dimensional phase-contrast images of the live mouse retina were created using multiple two-dimensional cross-sectional image slices through the retina. For the data presented here, each cross-sectional phase-contrast slice resulted from five images of 100 or 200 transverse pixels, acquired over $25 \mathrm{~ms}$ or $50 \mathrm{~ms}$, respectively. The approach offered clear identification of motion regions at different depths, including flow in the retinal microvasculature and in the choroidal vessels.

Conclusions. Phase-contrast OCT enables three-dimensional visualization of retinal and choroidal vasculature in vivo. (Invest Ophthalmol Vis Sci. 2008;49:5055-5059) DOI:10.1167/iovs.071627

$\mathrm{O}$ ptical coherence tomography (OCT) uses an interferometry imaging technique capable of producing cross-sectional reflectance images. This form of optical histology is ideal for imaging laminar structures such as the retina. ${ }^{1}$ High-speed developments in OCT systems have improved the ability to acquire large volumetric image sets and to increase the opportunities for additional image-contrast techniques based on the motion of the scatterers.

From the ${ }^{1}$ Department of Applied Physics and the ${ }^{2}$ Beckman Institute, Division of Biology, California Institute of Technology, Pasadena, California; and the ${ }^{3}$ Department of Ophthalmology, University of California and Veterans Affairs Medical Center, San Francisco, California.

Supported by That Man May See, the Hellman Foundation, the Lincy Foundation, Research to Prevent Blindness, and National Eye Institute Core Grant EY02162.

Submitted for publication December 18, 2007; revised February 17 and June 6, 2008; accepted September 15, 2008.

Disclosure: J. Fingler, P; C. Readhead, None; D.M. Schwartz, P; S.E. Fraser, $\mathrm{P}$

The publication costs of this article were defrayed in part by page charge payment. This article must therefore be marked "advertisement" in accordance with 18 U.S.C. $\$ 1734$ solely to indicate this fact

Corresponding author: Daniel M. Schwartz, Beckman Vision Center, UCSF School of Medicine, 10 Koret Way, Room K-301, San Francisco, CA 94143-0730; schwartz7@mindspring.com.
The interferometric information offered by OCT imaging can be used to determine the intensity of reflections from different depths in the retina at resolutions of a few microns, but it can also yield finer spatial information from the phase of the signals. In fact, measured phase changes can be used to extract nanometer scale motions of the reflections along the imaging (axial) direction. High-speed Doppler OCT techniques have demonstrated the capability of imaging the component of flow along the imaging direction (axial flow) using phase changes $^{2,3}$ and other Doppler techniques, ${ }^{4}$ but the transverse component of the flow is not visualized by such approaches. This is unfortunate because in the retina most of the flow is transverse to the imaging direction. We have developed a phase-contrast method for visualization of transverse flows, ${ }^{5}$ making it well suited for retinal vascular imaging using OCT. In our approach, the variance of the phase is used to identify locations of mobility within the OCT cross-sectional reflectance image, allowing us to visualize both the Brownian motion of scatterers and the transverse motion of scatterers moving within the blood. Here we report results of this novel phase-contrast OCT system demonstrating its ability to visualize retinal and choroidal vessels in the living mouse.

\section{Methods}

A high-speed spectral domain optical coherence tomography (SDOCT) system was developed for mouse retinal imaging in this experiment (Fig. 1). In this system, the imaging rate is determined by the readout rate of the spectrometer. The rate of acquisition for each axial scan (A-scan) is $25 \mathrm{kHz}$, which results in the acquisition of a 250-transverse pixel cross-sectional image in $10 \mathrm{~ms}$, approximately 25 to 100 times faster than conventional OCT imaging systems.

All procedures used in this study were performed in accordance with the ARVO Statement for the Use of Animals in Ophthalmic and Vision Research. In vivo imaging was performed on young adult B6D2F1 mice, anesthetized with a ketamine and xylazine solution containing $0.1 \mathrm{~mL}$ xylazine hydrochloride injection (Xylaject; Phoenix Pharmaceuticals, St. Joseph, MO) and $0.3 \mathrm{~mL}$ ketamine hydrochloride injection (Ketaject; Phoenix Pharmaceuticals) in $4 \mathrm{~mL}$ sterile phosphate-buffered saline (PBS). After anesthesia, the pupils were dilated with $1 \%$ tropicamide. The sample arm of the interferometer contained a contact lens composed of a glass coverslip with saline solution. This lens was applied to the mouse cornea before imaging to reduce ocular aberrations and to maintain corneal hydration. The animals were secured in a holder to align their eyes within the imaging system after the application of the contact lens. An external focusing lens of focal length $18 \mathrm{~mm}$ was applied to compensate for the removal of the corneal focusing. Transverse resolution was determined by the spot size of the focused light in air, which was approximately $11 \mu \mathrm{m}$. Axial resolution of the system in tissue was determined by the superluminescent diode (centered at $840 \mathrm{~nm}$ with a bandwidth of $50 \mathrm{~nm}$ ); resolution was approximately $6 \mu \mathrm{m}$.

SDOCT acquires reflectance information in axial scans called Ascans. Each A-scan contains the magnitude and location of all reflections along the full depth of the retina for a single location. Through collecting multiple A-scans over a linear set of neighboring positions, a cross-sectional reflectance image through the retina (B-scan) is created. Collecting a set of neighboring B-scans (cross-sections) creates a three- 


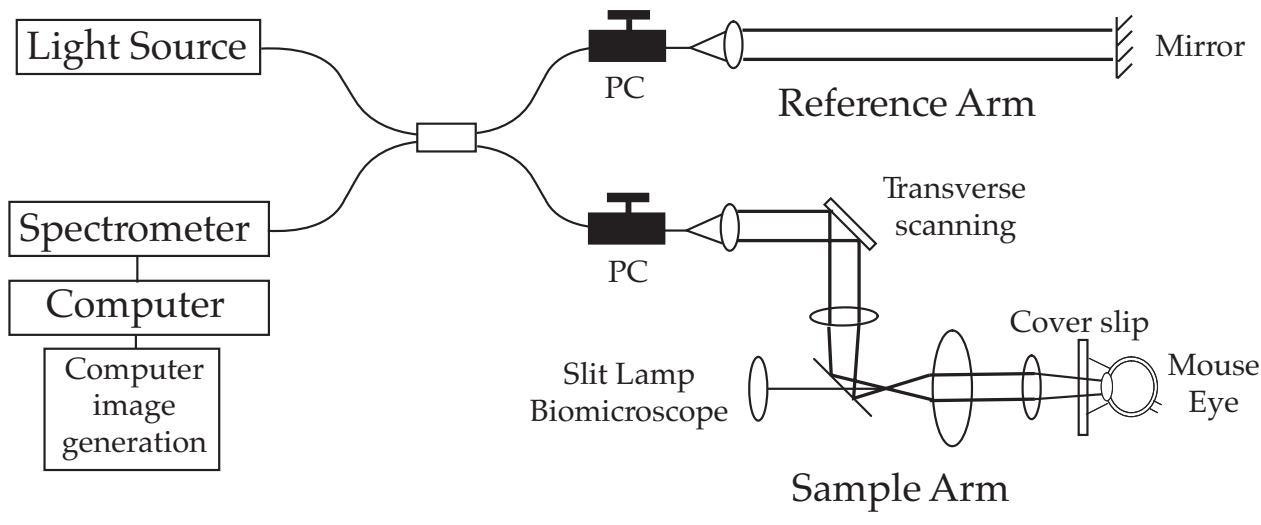

FIGURE 1. Schematic of fiber optic mouse retinal SDOCT system adapted from a human retinal imaging system. An additional focusing lens is added to the human retinal system to provide external focusing onto the retina of the coverslip covered mouse eye. PC, polarization controller. dimensional (volumetric) OCT image. To eliminate the curvature present in the cross-sectional images the mouse retina, the images were computationally flattened (in all figures presented here except Fig. 2) by the vertical realignment of the individual A-scans.

Phase contrast is achieved through determining the differences between multiple-phase measurements of the same locations, separated in time. The time separation between the images used for the phase measurements determines the minimum velocity that can be visualized by this technique. If one were to compare sequential A-scans at the same location, there would only be a time separation of $40 \mu \mathrm{s}$; as a result, only the fastest flows in the retina would be visualized with this method or other phase-sensitive flow methods. ${ }^{3,4}$ If instead phase contrast is obtained by comparing sequential B-scans, the increased time separation between phase measurements permits the visualization of motions 100 to 1000 times slower than achievable with sequential A-scans. Most phase-sensitive flow methods can only achieve such increased sensitivity to slow motions at the expense of visualizing the fastest flows. The phase-contrast technique we have developed for mobility and transverse flow visualization ${ }^{5}$ uses the statistical variance of phase to identify locations of mobility over a wide dynamic range. With this technique, slow motions can be visualized using sequential B-scans without sacrificing the fast flow visualization capabilities.

\section{Results}

A cross-sectional OCT intensity image of an in vivo mouse retina is presented in Figure 2, ${ }^{6,7}$ created from 200 A-scans extending over a transverse scan range of approximately $2 \mathrm{~mm}$.

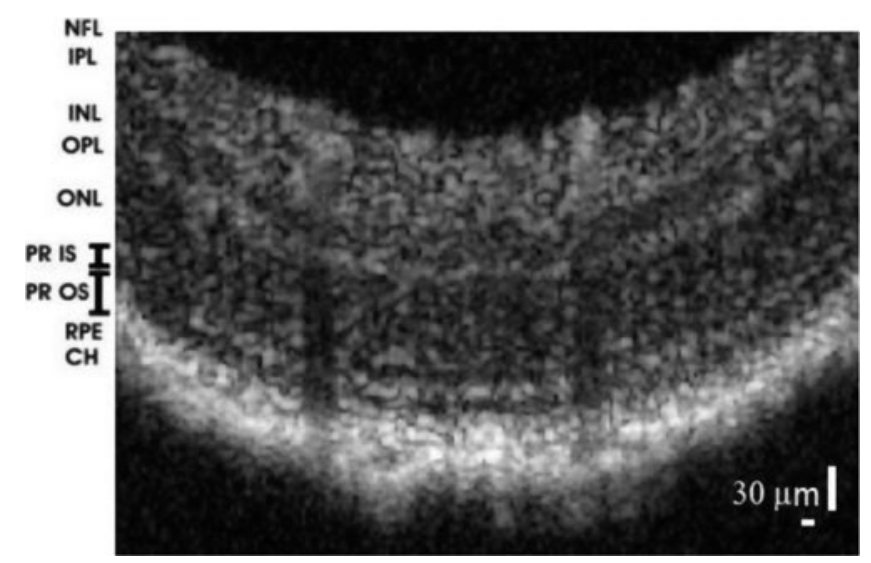

FIGURE 2. Cross-sectional OCT intensity image of mouse retina created using 200 A-scans acquired across a lateral extent of approximately $2 \mathrm{~mm}$. Retinal thickness and layering within the image is consistent with previously published results; approximate retinal layers are labeled (left $)^{6,7}$ As expected, given the small size of the mouse eye, the curvature of the retina is obvious within the images.
The intensity image is consistent with the expected results for adult mouse retina. ${ }^{6,7}$ Because of the thin structure of the retina, visualization of retinal structure is improved in all our images by using different scaling on the transverse and axial directions of the image. For all cross-sectional images, a linear scaling of 3:1 is used to make the retina appear thicker. Even with the curvature of the image from the small size of the mouse eye, major elements of the retinal anatomy are clear, including the nuclear and plexiform layers and the brightly reflecting RPE and choroid. The two darker vertical stripes within Figure 2 result from attenuation of the illumination by the light absorption of large blood vessels in the anterior portion of the retina. In all figures other than Figure 2, the images have been computationally flattened, eliminating the noticeable curvature of the mouse eye.

OCT intensity and phase-contrast cross-sectional images for the mouse retina in vivo are presented in Figure 3, created from five successive B-scans acquired in a total of $50 \mathrm{~ms}$. In these computationally flattened images, the intensity image identifies two large regions of retinal blood flow in the anterior retina as a region of increased scattering and by the darker vertical lines caused by the light absorption of the blood. The phase-contrast image is able to more clearly define these major blood vessels by their large phase variance. The phase-contrast image shows an elongation of the domain of phase variance into the depth of the retina because the light that contributes to the images of the deeper layers must pass through the moving blood in these major vessels and thus picks up phase variance. The smaller speckles that appear within the rest of the retinal region reflect the presence of microvasculature within the cross-sectional segment of the retina, which will be more fully explored in the later figures. In addition, the phasecontrast image shows significant signal from the choroidal region.

To permit a direct comparison of the regions of phase contrast with retinal structure, an overlay image of the same
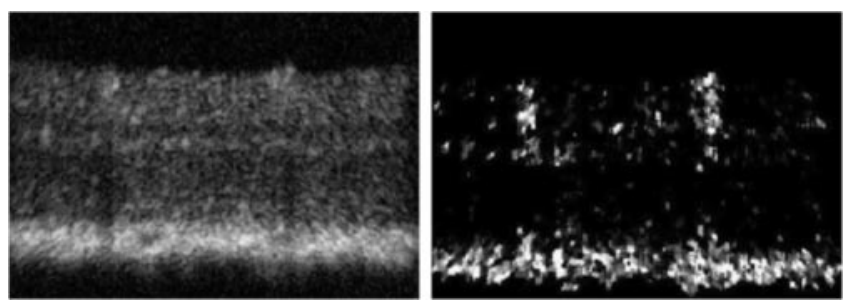

Figure 3. Averaged OCT intensity image (left) and phase-contrast image (right) of the mouse retina after computational retinal flattening. Both images were created from the same OCT data acquired in $50 \mathrm{~ms}$ from five B-scans, each consisting of 200 A-scans extending over a lateral extent of approximately $2 \mathrm{~mm}$. 


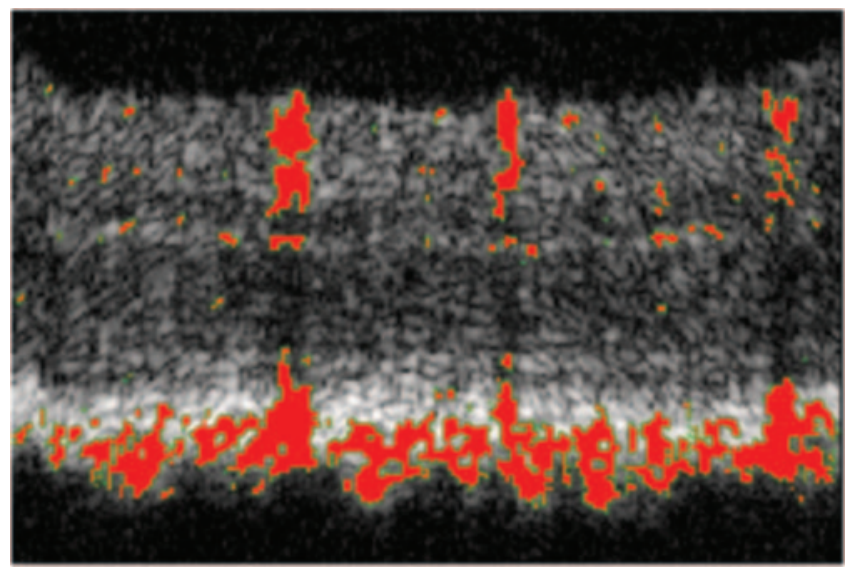

FIGURE 4. Overlay of phase-contrast image and OCT intensity image. Phase contrast is presented in red for signals above a phase-contrast threshold of 1 radian $^{2}$. The images are created from the same set of five B-scans acquired in $50 \mathrm{~ms}$, with each B-scan composed of 200 A-scans extending over $2 \mathrm{~mm}$.

data was created combining the phase-contrast and OCT intensity images (Fig. 4). ${ }^{2}$ Superposition of the data is straightforward because the same scans were used to create the intensity and phase images. As in Figure 3, the intensity and phase images were calculated from five B-scans acquired in a total of $50 \mathrm{~ms}$. Resolution of the intensity image averaged over the five frames shows that major motions of the retina did not occur during the 50-ms acquisition time. Regions of phase contrast calculated over a threshold level of $1 \operatorname{radian}^{2}$ are rendered in red and overlaid on top of the intensity image. There is some phase contrast in the RPE, but this is directly below major surface vessels; therefore, this signal is an artifact resulting from the two passages of the imaging light through the rapidly moving blood. Most phase contrast within the posterior segment of the retina corresponds to regions in the choroid, below the RPE.

Unlike the intensity image, which offers resolution in the micrometer range, phase-contrast techniques are sensitive to motions into the nanometer range. Our protocol measures phase changes between successive B-scans (a time separation of $10 \mathrm{~ms}$ ) that improve the ability to observe regions of very slow flow, but it also increases the susceptibility of the measurement to slight motion of the eye. Axial motion can be compensated by analyzing the simultaneous phase changes of all reflectances within the retina and subtracting any bulk motions. However, transverse motion during the 50-ms phasecontrast image acquisition is not as easily compensated; it will create background noise in the phase-contrast images that must be removed. If the transverse motion of the eye is at a frequency slower than any one image acquisition, the entire phase-contrast image will experience approximately the same transverse motion noise. Thus, using the statistics of the nonzero intensity pixels of the entire phase-contrast image, transverse motion noise was approximated and subtracted from the image.

Three-dimensional OCT data sets were acquired across the retina over a transverse scan area of approximately $2 \mathrm{~mm} \times 2$ $\mathrm{mm}$ by collecting 51 neighboring B-scans, each containing 200 A-scan locations. To create the two-dimensional images presented here from the three-dimensional data sets, a projection image was created for both the OCT intensity and the phasecontrast data sets by summing over the entire depth of the retina (Fig. 5). As expected, the projection of the OCT intensity data set (Fig. 5, left) is consistent with reflectivity fundus images produced with scanning laser ophthalmoscopes, with
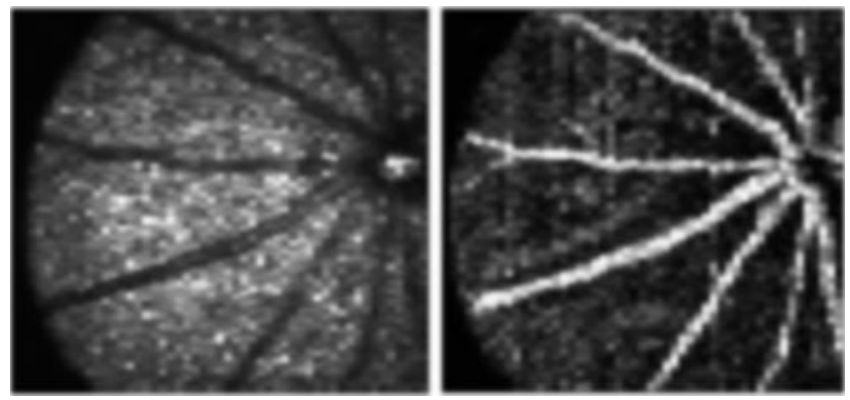

FIGURE 5. Transverse intensity (left) and phase-contrast (right) projection images summed over the full depth of the mouse retina. Within the phase-contrast projection image, the major retinal vasculature is intense; the choroidal vessels are more dimly rendered. Image size is $200 \times 51$ pixels over approximately $2 \mathrm{~mm} \times 2 \mathrm{~mm}$.

the major blood vessels appearing dark because of the blood absorption of the light. The phase-contrast projection (Fig. 5, right) highlights the major blood vessels of the retina as bright regions attributed to the significant motion of the blood, which gives a large phase signal. The representations in Figure 5 have two significant limitations: first, they are pixilated as a result of chosen image acquisition parameters that maximized the scan area imaged in the data acquisition time; second, the data were summed over the entire depth of the retina, making it difficult to determine the origin of the motions that give rise to the low-intensity phase features in the right panel.

To offer better depth discrimination, projection images were created that were summed over only the posterior or the anterior sections of the retina. The intensity image from only the anterior (Fig. 6, left) or posterior (Fig. 6, right) sections of the retina show the expected features. The anterior section intensity image is dominated by reflections from the anterior surface of the retinal blood vessels and the optic nerve head. The posterior intensity projection image is dominated by the reflections of the RPE layer and the choroid, with shadows identifying locations where the anterior reflections and light absorption of the major retinal vessels reduced the reflected light intensity.

Phase-contrast projection images summed over the anterior and posterior regions of the data set are shown in Figure 7 . The anterior phase-contrast image shows high intensity from the major vessels and some indication of signal from the surface microvasculature (Fig. 7, left, arrows), but this is obscured by the pixilation and limited time of data collection. The posterior phase-contrast image shows a lower intensity signal from the major vessels because the light reflected from deeper layers must pass through the major vessels to and from their site of reflection in the posterior retina. Because the blood is moving
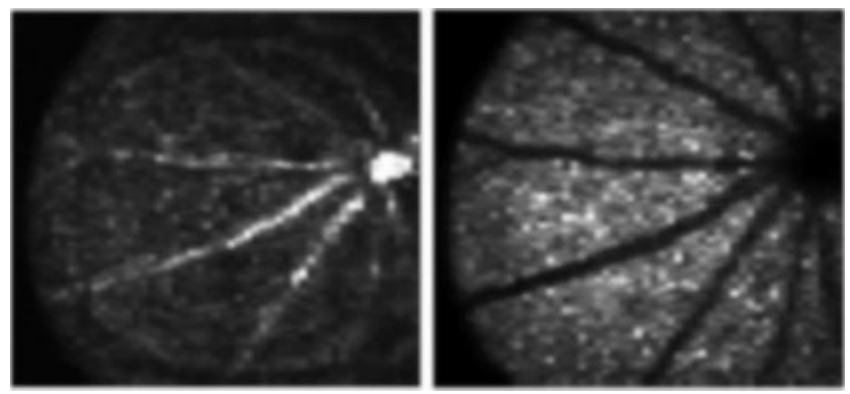

FIGURE 6. Transverse intensity projection images summed over the anterior (left) and the posterior (right) segments of the retina. Both images are created from the same data set as in Figure 5, composed of $200 \times 51$ pixels over approximately $2 \mathrm{~mm} \times 2 \mathrm{~mm}$. 

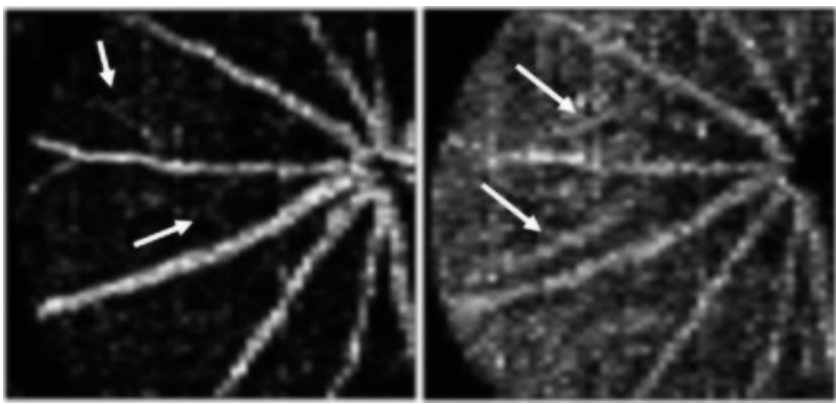

FIGURE 7. Phase-contrast projection images summed over the anterior (left) and posterior (right) segments of the retina. Both images are created from the same data set as in Figure 5, composed of $200 \times 51$ pixels over approximately $2 \mathrm{~mm} \times 2 \mathrm{~mm}$. Arrows: locations of phase contrast in a given depth section of the three-dimensional data set resulting from surface microvasculature (left) and choroidal vasculature (right).

in the major vessels, the transit through the vessels adds some phase variance. In addition, there are features that can be identified as choroidal vessels in the posterior section image (Fig. 7, right, arrows)

To improve visualization of the retinal microvasculature and reduce pixilation artifacts, three-dimensional OCT data were acquired from the in vivo mouse retina over a smaller region (1 $\mathrm{mm} \times 1 \mathrm{~mm})$. Two-dimensional projection images $(100 \times 50$ pixels) were created from the three-dimensional data sets. Figure 8 presents an intensity projection image summed over the entire depth of the retina (left), and a phase-contrast projection image was summed over only the anterior section of the retina (right). Superficial retinal microvasculature is observed within the phase-contrast projection image.

Phase-contrast OCT images require a moving scatterer with a significant reflection within a given pixel to produce a signal. Although flow may occur within the microvasculature at most times, transparent plasma within the vessels does not produce a significant reflection whereas a blood cell would. Blood cells within the microvasculature are spaced from one another and move slowly; thus, a phase-contrast image taken at any moment in time would show the presence of the moving scatterers as individual locations of phase contrast rather than reveal the entire microvasculature. Mapping the microvasculature requires multiple phase-contrast images acquired successively to capture these motions. Figure 9 shows four sequential phase-contrast images separated in time by 1.3 seconds over the same scan area presented in Figure 8 . Each of the projection phase images $(100 \times 50$ pixels $)$ was summed over the
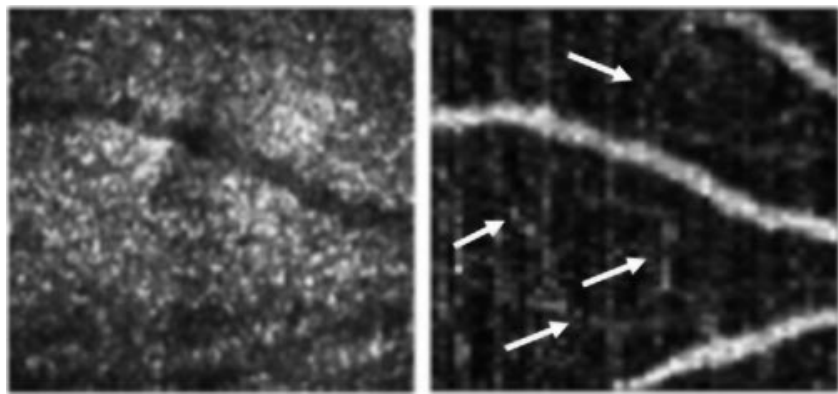

FIGURE 8. Transverse intensity projection images summed over the entire retina (left) and phase contrast summed over the anterior section of the retina $(r i g h t)$. Both images are created from the same data set, $200 \times 51$ pixels acquired in 2.6 seconds, extending over approximately $1 \mathrm{~mm} \times 1 \mathrm{~mm}$. Arrows: some of the microvasculature appears within the phase-contrast projection image.
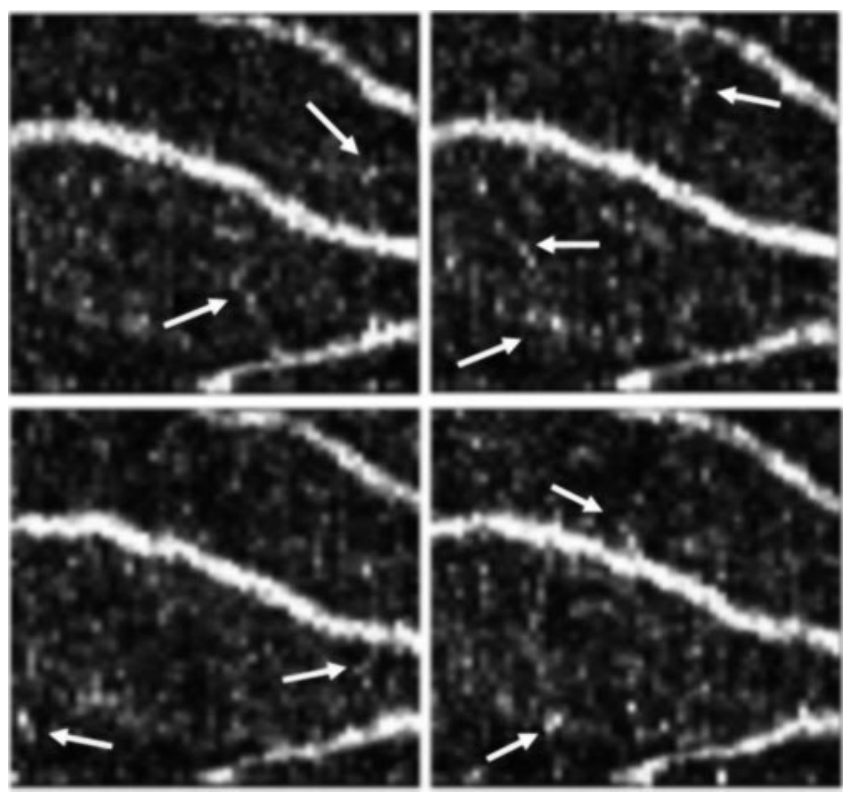

FIGURE 9. Phase-contrast projection images created from four successively acquired three-dimensional OCT data sets, separated in time by 1.3 seconds. Each image was acquired over an area of approximately 1 $\mathrm{mm} \times 1 \mathrm{~mm}$ and interpolated to $100 \times 100$ pixels. Arrows: locations of moving scatterers within the vasculature of one of the frames but not all four.

anterior section of the retina. Note that each captures different regions of the microvasculature (arrows), suggesting that by combining phase-contrast data from multiple images, a more complete image of the microvasculature can be generated.

To test whether the visualization of retinal microvasculature is improved by increasing the number of phase-contrast images acquired over the same transverse area at different time points and averaging, we collected phase-contrast image from two sets of four images, each separated by 1.3 seconds (Fig. 10). Each of the projection images was interpolated to $100 \times 100$ pixels. To minimize potential artifacts from eye motion and to improve vascular visualization, the data sets were acquired in two sets of four images, with the primary scan direction for the two sets perpendicular to each other. This would make any transverse motion noise of the sample impact resolution in a different direction. The final projection of the eight images results in an improved image of the microvasculature (Fig. 10). Each cross-sectional image used to create Figures 9 and 10 had

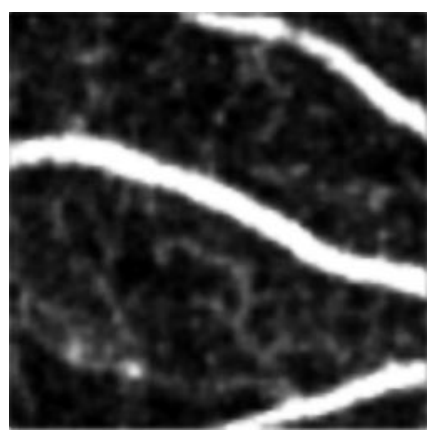

FIGURE 10. Mean anterior retinal phase-contrast projection image created from eight separate projection images after transverse alignment. Each image was acquired over approximately $1 \mathrm{~mm} \times 1 \mathrm{~mm}$ in 1.3 seconds and was extrapolated to $100 \times 100$ pixels. Gamma correction is used to enhance retinal microvascular visualization within the phasecontrast image. 
acquisition times of $25 \mathrm{~ms}$, demonstrating less sensitivity to sample motion than images of larger acquisition times and, in this case, yielding minimal transverse motion noise. The two groups of four data sets used to create the image in Figure 10 were separated by at least 1 minute; any transverse motion drift of the eye between image sets was compensated by computational alignment of the data sets before further processing.

\section{Discussion}

We have previously developed methods to enhance the contrast of structural OCT images based on the small motion of mobile scatterers within the sample. ${ }^{5}$ Phase contrast was observed in images for motions smaller than $50 \mathrm{~nm}$ for a variety of sample motions, including flow in any direction and diffusional Brownian motion. Here we extend these initial observations to the visualization of the retinal and choroidal vasculature of the live mouse. Three-dimensional phase-contrast images of the mouse retina were created using multiple twodimensional cross-sectional phase-contrast slices through the retina. With the demonstrated imaging system, each crosssectional phase-contrast slice with 100 or 200 transverse pixels was acquired in $25 \mathrm{~ms}$ or $50 \mathrm{~ms}$, respectively. Segmentation of the three-dimensional phase-contrast data allowed for the identification of phase-contrast regions at different depths, including the flow associated with microvasculature in the retina and the choroidal vessels. Because of the discrete nature of the scatterers in the microvasculature, complete phase-contrast images of the microvasculature required multiple imaging sets separated in time.

The current imaging protocol was optimized for determining regions of flow, even if transverse. The advantages that make it capable of detecting transverse flow negate its ability to make quantitative measures of flow. Once the three-dimensional structure of the vasculature is identified with this method, other flow methods previously described ${ }^{2,3}$ could be used with the geometric information to quantify flow within the vessels.

Because our technique requires the acquisition of multiple images to calculate phase contrast, it is critically dependent on the acquisition speed of the OCT system. The OCT system we used offered a minimum acquisition time of $25 \mathrm{~ms}$ for 100 transverse pixels. Improvements in the OCT instrumentation to increase acquisition speed can reduce the total imaging time and should decrease the noise effects created by microsaccadic motion on phase-contrast imaging. In addition, such improvements in imaging speed would increase the total image size. Even with a technological improvement in acquisition speed by a factor of 10 , there is enough flexibility to optimize the phase-contrast imaging parameters to reduce the effects of the bulk sample motion while still visualizing the phase contrast of the individual scatterers within the sample.

We are planning to test the ability of the phase-contrast SDOCT to visualize Bruch membrane lipid and choroidal neovascularization in patients. No specific problems are anticipated for the transition of this method to apply to human retinal imaging. Experimental and clinically available OCT systems have demonstrated full penetration of the retina and partial penetration of the choroid using 800-nm light. For deeper penetration of the choroid, longer wavelength light of approximately $1 \mu \mathrm{m}$ has demonstrated excellent choroidal imaging capabilities. ${ }^{8}$ Finally, the method of vascular visualization used here does not rely on any particular wavelength of light for its performance.

\section{Acknowledgment}

The authors thank Carl Zeiss Meditec for generously providing technical and material assistance in the development of the imaging system.

\section{References}

1. Huang D, Swanson EA, Lin CP, et al. Optical coherence tomography. Science. 1991;254:1178-1181.

2. White BR, Pierce MC, Nassif N, et al. In vivo dynamic human retinal blood flow imaging using ultra-high-speed spectral domain optical coherence tomography. Opt Express. 2003;11:3490-3497.

3. Leitgeb RA, Schmetterer L, Hitzenberger CK, et al. Real-time measurement of in vitro flow by Fourier-domain color Doppler optical coherence tomography. Opt Lett. 2004;29:171-173.

4. Bachmann H, Villiger ML, Blatter C, Lasser T, Leitgeb RA. Resonant Doppler flow imaging and optical vivisection of retinal blood vessels. Opt Express. 2007;15:408-422.

5. Fingler J, Schwartz D, Yang C, Fraser SE. Mobility and transverse flow visualization using phase variance contrast with spectral domain optical coherence tomography. Opt Express. 2007;15:1263612653.

6. Srinivasan VJ, Ko TH, Wojtkowski M, et al. Noinvasive volumetric imaging and morphometry of the rodent retina with high speed, ultrahigh-resolution optical coherence tomography. Invest Ophthalmol Vis Sci. 2006;47:5522-5528.

7. Ruggeri M, Wehbe $\mathrm{H}$, Jiao $\mathrm{S}$, et al. In vivo three-dimensional highresolution imaging of rodent retina with spectral-domain optical coherence tomography. Invest Ophthalmol Vis Sci. 2007;48:18081814 .

8. Povazay B, Hermann B, Unterhuber A, et al. Three-dimensional optical coherence tomography at $1050 \mathrm{~nm}$ versus $800 \mathrm{~nm}$ in retinal pathologies: enhanced performance and choroidal penetration in cataract patients. J Biomed Opt. 2007;12:041211. 